\title{
Automated evaluation and normalization of immunohistochemistry on tissue microarrays with a DNA microarray scanner
}

\author{
Wolfgang Haedicke1, Helmut H. Popper², Charles R. Buck, \\ and Kurt Zatloukal ${ }^{1,2}$ \\ ${ }^{1}$ Oridis Biomed, and ${ }^{2}$ University of Graz, Graz, Austria
}

BioTechniques 35:164-168 (July 2003)

Hundreds of tissue samples may be assembled in a tissue microarray format for simultaneous immunostaining assessment of protein expression profiling. A DNA microarray two-color laser scanner was used for automated analysis of tissue microarray indirect immunofluorescence. On sections from both a human lung adenocarcinoma and a squamous cell carcinoma tissue microarray, fluorescence intensity for two epidermal growth factor receptors (EGFR and c-erbB2) correlates with diagnostic pathologist assessment, indicating that immunohistochemistry quantitation can be achieved. Importantly, double-label indirect immunofluorescence detection with the cDNA scanner demonstrates that one reference antigen can normalize tumor marker immunosignal for the cellular content of tissue microarray tissue cores. Therefore, DNA microarray scanners and associated image analysis software provide general and efficient analysis of tissue microarray immunostaining, including estimation of specific protein expression levels.

\section{INTRODUCTION}

High-throughput DNA microarray screening has led to the identification of large numbers of deregulated genes with potential utility as new diagnostic and therapeutic targets. Subsequent examination of expression patterns of the encoded proteins in normal and diseased tissues is critical for the determination of the disease relevance of these candidate targets. Immunohistochemistry localization of the deregulated gene products in human tissues can provide this information, but a conventional target-by-target, tissue-by-tissue approach is not practicable for large numbers of deregulated genes.

Tissue microarrays enable representation on one microscope slide of hundreds of tissue cores and thereby provide a corresponding high-throughput approach to this validation step (1). In addition to a broad representation of normal and/or diseased human tissues, tissue microarrays minimize depletion of precious human tissue resources (2). However, the evaluation of immunohistochemistry on each tissue microarray slide generally requires manual analysis and tracking of hundreds of samples. Automated immunohistochemistry image analysis systems may be modified for tissue microarrays, but these systems are expensive and not widely available.

There is a need for efficient methods to normalize immunohistochemistry signals for cellular content because specific protein expression levels may reflect biological relevance. This issue has particular importance with tissue microarrays because the tissue core represents a small fraction of the tissue sample employed for diagnosis. Double-label immunohistochemistry with a two-color detection system is a powerful method to achieve normalization for cellular content. DNA microarray scanners, available in core facilities at most research institutions, specifically and simultaneously detect two fluorophores, and the associated image analysis software processes the fluorescent signals. We therefore employed a dualwavelength DNA array scanner and double indirect immunofluorescence to normalize target gene expression signal to tumor cell content. This approach provides signal intensity data that indicate qualitative and quantitative aspects of protein expression as evaluated with indirect immunofluorescence on several tissue microarrays.

\section{MATERIALS AND METHODS}

\section{Tissue Microarrays}

Tissues from The Institute of Pathology, University of Graz, were fixed in buffered $4 \%$ formalin. Tissue microarrays were constructed using a manual tissue-arraying instrument (Beecher Instruments, Sun Prairie, WI, USA) $(1,3,4)$. Multiple tissue cores from each donor block were included in each tissue microarray.

\section{Immunostaining}

Five-micron tissue microarray sections on Superfrost ${ }^{\mathrm{TM}}$ Plus slides (Roth, Karlsruhe, Germany) were deparaffinized and treated with $1 \% \mathrm{H}_{2} \mathrm{O}_{2}$ in PBS, and the antigens were retrieved with microwaves $(5 \mathrm{~min}$ at $800 \mathrm{~W}, 20$ min at $300 \mathrm{~W}$, in $10 \mathrm{mM}$ citrate buffer, pH 6.0). Sequential 30-min, room temperature antibody incubations were followed with standard washing steps. Stained slides were dehydrated and coverslipped with Fluoromount (Serva, Heidelberg, Germany), and digital images were obtained with a laser scanning microscope (LSM510; Zeiss, Jena, Germany).

\section{Automated Evaluation of Tissue Microarrays}

Tissue microarray immunohistochemistry .tif images from $10-\mu \mathrm{m}$ GMS418 scans (Affymetrix, Santa Clara, CA, USA) were analyzed with ImaGene 4.1 software. A grid adjusted to accommodate the tissue microarray tissue cores provided signals from which local background was subtracted according to the software instructions. Small tissue defects did not eliminate cores from analysis, as the normalized procedure eliminated this source of artifact. Relative signal intensity and specificity were confirmed with a fluorescence microscope. Microsoft ${ }^{\circledR}$ Excel $^{\circledR}$ or SPSS ${ }^{\circledR}$ was used for statistical analysis. 
Table 1. Tissue Microarray Tissue Core Tumor Marker Signal Intensity Obtained with a DNA Microarray Scanner and Normalized for Tissue Epithelial Cell Content

\begin{tabular}{|ccccc|}
\hline $\begin{array}{c}\text { Tissue } \\
\text { Core }\end{array}$ & $\begin{array}{c}\text { Raw } \\
\text { c-erbB2 Signal }\end{array}$ & $\begin{array}{c}\text { Initial } \\
\text { Rank }\end{array}$ & $\begin{array}{c}\text { Normalized } \\
\text { c-erbB2 Signal }\end{array}$ & $\begin{array}{c}\text { Normalized } \\
\text { Rank }\end{array}$ \\
\hline A & 29,161 & 4 & 4.88 & 1 \\
B & 41,500 & 2 & 4.23 & 2 \\
C & 43,000 & 1 & 3.49 & 4 \\
D & 39,000 & 3 & 4.03 & 3 \\
\hline
\end{tabular}

\section{RESULTS AND DISCUSSION}

Indirect immunofluorescence detection of multiple antigens on several tissue microarrays using a DNA microarray scanner was sensitive and reproducible. To provide an initial validation for this approach, a well-established immunohistochemistry method for distinction of epithelial tumors was employed. Cytokeratin expression with the pan-cytokeratin antibodies AE1/ AE3 (Dako, Glostrup, Denmark) on a small tumor tissue microarray demonstrated at least 10-fold higher levels of these isoforms in carcinoma tissue cores compared with sarcoma cores on the same array, as expected $(P<0.001$, two-sided $t$ test; data not shown) (5). Independent labeled secondary antibodies demonstrated that the apparent cytokeratin signal detected in sarcomas was background. For single-fluorophore indirect immunofluorescence, this should be taken into consideration and may be eliminated by setting the background threshold manually. For double-label indirect immunofluorescence analysis, the background was similar with both secondary antibodies and therefore did not adversely affect the data quantitation and analysis.

\section{Normalization and Quantitative Evaluation of Tissue Microarray Indirect Immunofluorescence}

The variability of a tumor marker immunohistochemistry signal due to differential tumor cell content is a limiting factor for comparison of signals from different tissue microarray tumor cores. We therefore tested the possibility that double-label indirect immunofluorescence measured with a two-color DNA microarray scanner could normalize tissue microarray tissue core signals. Sections from two lung cancer

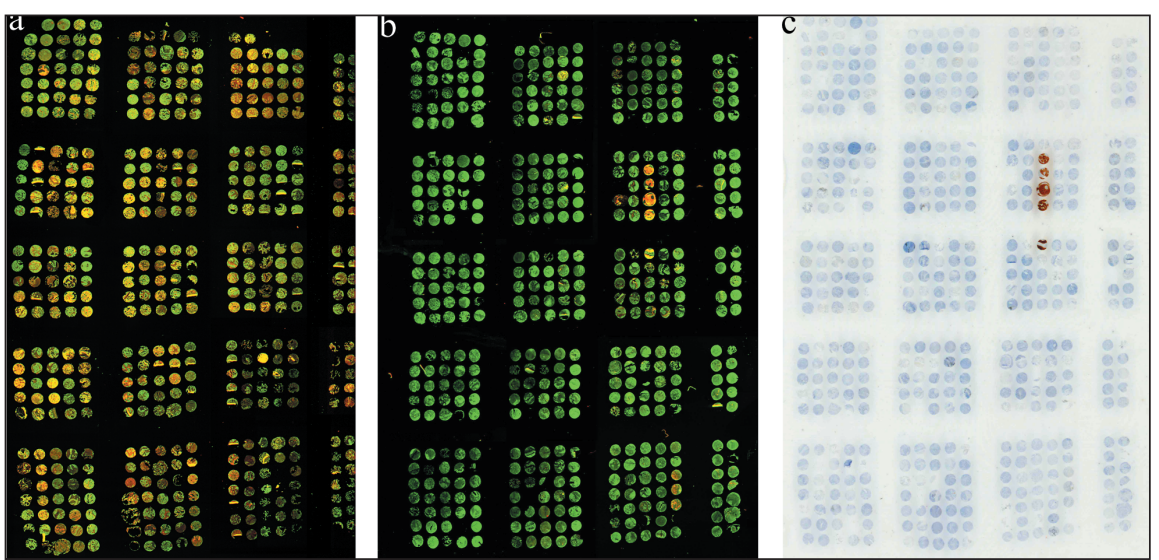

Figure 1. Double immunostaining on tissue microarrays. (a) Double-label indirect immunofluorescence of a lung adenocarcinoma tissue microarray stained with antibodies to the pan cytokeratin marker $\mathrm{AE} 1 / \mathrm{AE} 3$ (green) and to the tumor marker c-erbB2 (red), scanned with a DNA microarray scanner. (b) Double-label indirect immunofluorescence of a lung squamous cell carcinoma tissue microarray stained and scanned as in panel a. (c) The same squamous cell carcinoma array as in panel b, stained with the cerbB2 diagnostic kit HercepTest ${ }^{\mathrm{TM}}$ (Dako). The weakly positive cores in panel $\mathrm{b}$ are negative with this diagnostic immunohistochemistry procedure because of the lower sensitivity of the test. 
tissue microarrays were double-stained with antibodies to the tumor markers cerbB2 (Dako), epidermal growth factor receptor (EGFR) (Santa Cruz Biotechnology, Santa Cruz, CA, USA), or CD44 variant 6 (CD44v6; Bender MedSystems, Vienna, Austria) and anti-cytokeratin $(5,6)$. Cytokeratin sig- nal normalized each tissue core for the amount of epithelial carcinoma cells represented. Lung adenocarcinoma TMA (75 cases, 476 cores) and lung squamous cell carcinoma tissue microarray (67 cases, 479 cores) sections were labeled with c-erbB2 or EGFR polyclonal and cytokeratin monoclonal
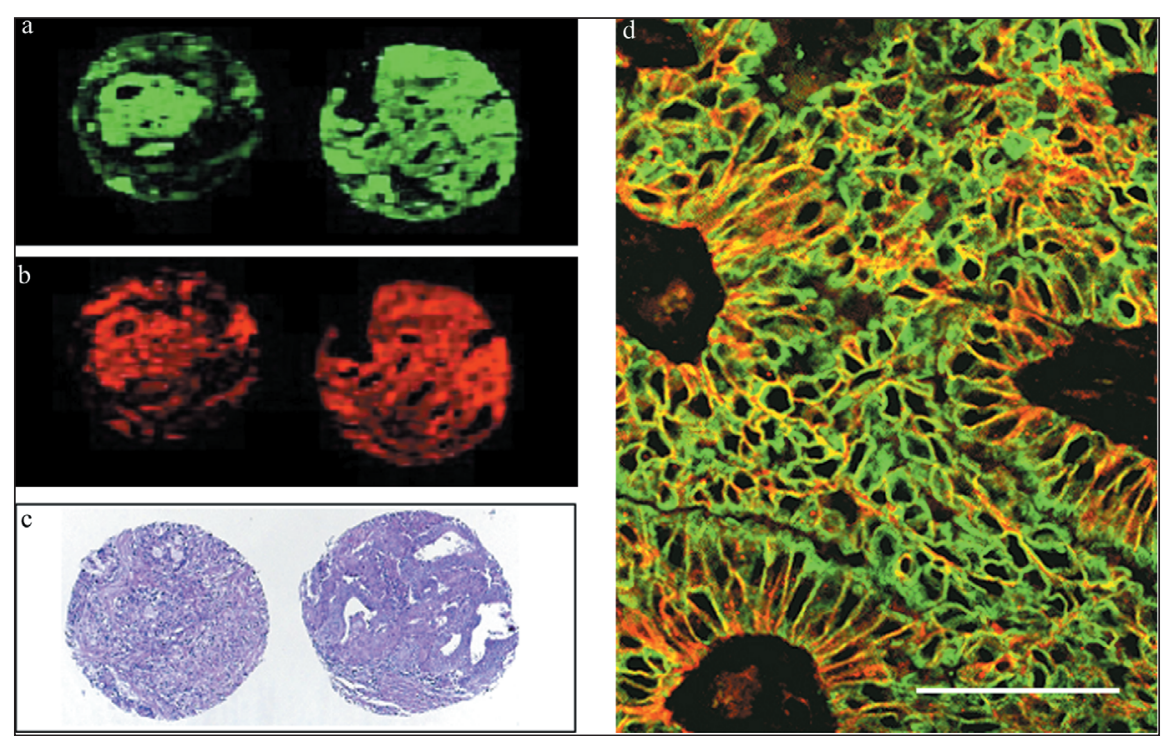

Figure 2. Effects of normalization on tissue microarray tumor marker indirect immunofluorescence signal level intensity. (a) The tumor cell marker c-erbB2 on two representative cores from the lung adenocarcinoma tissue microarray. (b) The carcinoma cellular content is indicated by staining with a pan cytokeratin antibody on the same cores in the same section as shown in panel a. (c) Micrograph of these same two tissue cores from a different section of the array stained with hematoxylin and eosin. (d) Microscopic image of double-label indirect immunofluorescence on tissue microarray tissue core B. The cellular detail and co-localization of the tumor marker c-erbB2 (red) and the epithelial cell marker AE1/AE3 (green) are illustrated. The tissue cores in panels a-c have a diameter of approximately 0.6 $\mathrm{mm}$; the scale bar in panel $\mathrm{d}$ is $25 \mu \mathrm{m}$.

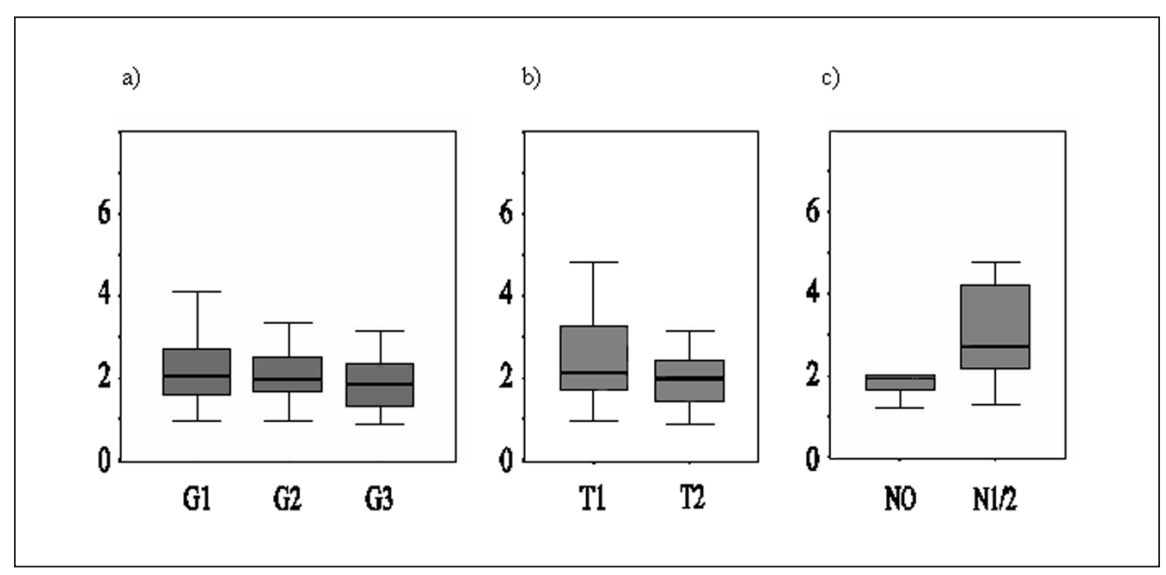

Figure 3. Normalized tumor marker indirect immunofluorescence signal intensity compared to diagnostic parameters of the tissue, lymph node, distant metastasis system. Correlation of CD44v6 to tumor differentiation (a; stages G1-G3), tumor stage (b; T1 or T2), and lymph node metastatic stage (c; $\mathrm{N} 0$ or N1/2). Normalized fluorescence intensity is provided on the y axis (quotient of c-erbB2 and AE1/AE3 signal intensities in arbitrary units). The difference in expression levels of CD44v6 in N0 versus N1/2 tumors is statistically significant $(P<0.5)$. antibodies, and the lung adenocarcinoma tissue microarray sections were labeled with the CD44v6 monoclonal and cytokeratin $8 / 18$ polyclonal antibodies (Figure 1).

Each tumor marker signal detected with a $\mathrm{Cy}^{\mathrm{TM}} 3$-conjugated secondary antibody was normalized by division with the keratin signal detected with a Cy5conjugated secondary antibody. Normalization of the 5-7 cores from each tumor reduced variability in tumor marker expression between cores from the same tumor. Distinct c-erbB2 expression on the lung squamous cell carcinoma TMA was confined to just one of the 76 cases (Figure 1, b and c) $(7,8)$, and normalization reduced the variability by $33 \%$ [average coefficient of variation $(\mathrm{CV})$ reduced from 0.3 to $0.2 ; P<$ 0.01 , Student's $t$ test].

Conventional EGFR immunohistochemistry (graded negative/weakly positive/strongly positive) revealed 18 weakly positive and 26 strongly positive cores on the adenocarcinoma array (44 of 479), and 12 weakly positive cores on the squamous cell carcinoma array (of 476). Compared with DNA microarray scanner-derived normalized values for these cores, the weakly positive samples were significantly stronger than those judged as negative, and the strongly positive were significantly different from those judged weakly positive (each $P<0.01$, Student's $t$ test).

Table 1 provides the raw and normalized c-erbB2 signal intensity from four adjacent cores on the lung adenocarcinoma tissue microarray (two of these cores are shown in Figure 2, a and b). The intensity of c-erbB2 expression is greatest in core A tumor cells, despite the lowest absolute tumor marker signal for this core. The DNA microarray scanner analysis approach does not preclude detailed histological examination of immunostaining results, as indicated with histochemical and indirect immunofluorescence staining of these cores (Figure 2, c and d).

\section{Evaluation of Correlations with Clinicopathologic Parameters}

Correlation with clinical and diagnostic parameters would be a major indication for the automated evaluation of immunostaining on tissue microarrays. 
Therefore, we compared tumor marker indirect immunofluorescence signals obtained from the DNA array scanner on the lung adenocarcinoma tissue microarray to the diagnostic pathology tumor, lymph node, distant metastasis staging and grading system (9).

Normalized CD44v6 expression correlated with node metastasis, but not with tumor size or differentiation $(P<$ 0.05 ; Figure 3 ); consistent with the finding that CD44v6 expression levels correlate with the metastatic potential of cell lines (10) and higher recurrent distant metastases in CD44v6-positive non-small-cell lung carcinoma (11). However, a lack of correlation between metastatic stage and CD44v6 expression has also been reported (12).

Normalized c-erbB2 expression levels determined with indirect immunofluorescence on tissue microarrays did not correlate with tumor lymph node metastatic stage, as previously reported (13). On this lung adenocarcinoma tissue microarray, poorly differentiated (G3) tumors expressed significantly lower c-erbB2 levels than moderately and well-differentiated tumors (G1 and G2), and levels of this antigen were higher in stage T1 tumors than in T2 tumors $(P<0.05)$ (data not shown). Although c-erbB2 expression in nonsmall cell lung carcinoma has been correlated with a higher tumor stage and positive lymph node status, this study assessed only membranous staining, semiquantitatively (14). A clinicopathologic role of c-erbB2 in lung cancer remains controversial both with regard to potential disease involvement and to assessment methodology.

The expression of EGFR on this tissue microarray did not show any significant correlation with tumor, lymph node, distant metastasis parameters. Furthermore, expression of EGFR in these tissues correlated with neither cerbB2 nor CD44v6 expression (data not shown), which is in agreement with previous reports (15).

In conclusion, application of a DNA microarray scanner and analysis software for tissue microarray indirect immunofluorescence data provides an efficient and versatile tool for initial automated analysis of this protein expression profiling approach. Doublelabel indirect immunofluorescence normalization extends the power of this approach by reducing the effects of tissue variability.

\section{ACKNOWLEDGMENTS}

The technical expertise of Andrea Fuchsbichler, Iris Halbwedl, Peggy Samitch, and Andrea Sommersacher is gratefully acknowledged. Supported by the Austrian FFF fund (Oridis) and by the Austrian Science Fund (14759 to H.P. and S7401-MOB to K.Z.).

\section{REFERENCES}

1.Kononen, J., L. Bubendorf, A. Kallioniemi, M. Barlund, P. Schraml, S. Leighton, J. Torhorst, M.J. Mihatsch, et al. 1998. Tissue microarrays for high-throughput molecular profiling of tumor specimens. Nat. Med. 4:844-847.

2.Tschulik, A. and K. Zatloukal. 2001. The increasing importance of tumor and tissue banks in the light of genomic and proteomic research. Pathologe 22:310-315.

3.Hoos, A., M.J. Urist, A. Stojadinovic, S. Mastorides, M.E. Dudas, D.H. Leung, D. Kuo, M.F. Brennan, et al. 2001. Validation of tissue microarrays for immunohistochemical profiling of cancer specimens using the example of human fibroblastic tumors. Am. J. Pathol. 158:1245-1251.

4.Mucci, N.R., G. Akdas, S. Manely, and M.A. Rubin. 2000. Neuroendocrine expression in metastatic prostate cancer: evaluation of high throughput tissue microarrays to detect heterogeneous protein expression. Hum. Pathol. 31:406-414.

5.Schaafsma, H.E. and F.C. Ramaekers. 1994 Cytokeratin subtyping in normal and neoplastic epithelium: basic principles and diagnostic applications. Pathol. Annu. 29Pt1:21-62.

6.Fickert, P., M. Trauner, A. Fuchsbichler, C. Stumptner, K. Zatloukal, and H. Denk. 2002. Cytokeratins as targets for bile acid-induced toxicity. Am. J. Pathol. 160:491-499.

7.Pfeiffer, P., P.P. Clausen, K. Andersen, and C. Rose. 1996. Lack of prognostic significance of epidermal growth factor receptor and the oncoprotein p185HER-2 in patients with systemically untreated non-small-cell lung cancer: an immunohistochemical study on cryosections. Br. J. Cancer 74:86-91.

8.Tateishi, M., T. Ishida, T. Mitsudomi, S. Kaneko, and K. Sugimachi. 1991. Prognostic value of c-erbB-2 protein expression in human lung adenocarcinoma and squamous cell carcinoma. Eur. J. Cancer 27:1372-1375.

9.Sobin, L.H. (Ed.). 2002. TNM Classification of Malignant Tumours. John Wiley \& Sons, New York.

10.Carbognani, P., L. Spaggiari, A. Romani, P. Solli, A. Corradi, A.M. Cantoni, P.G. Petronini, A.F. Borghetti, et al. 1998. Expression of human CD44v6 in non-small-cell lung cancer. Eur. Surg. Res. 30:403-408.
11.Hirata, T., T. Fukuse, H. Naiki, S. Hitomi, and H. Wada. 1998. Expression of CD44 variant exon 6 in stage I non-small cell lung carcinoma as a prognostic factor. Cancer Res. 58:1108-1110.

12.Ramasami, S., K.M. Kerr, A.D. Chapman, G. King, J.S. Cockburn, and R.R. Jeffrey. 2000. Expression of CD44v6 but not E-cadherin or $\beta$-catenin influences prognosis in primary pulmonary adenocarcinoma. J. Pathol. 192:427-432

13.Volm, M., G. van Kaick, and J. Mattern. 1994. Analysis of c-fos, c-jun, c-erbB1, cerbB2 and c-myc in primary lung carcinomas and their lymph node metastases. Clin. Exp. Metastasis 12:329-334.

14.Kristiansen, G., Y. Yu, S. Petersen, O. Kaufmann, K. Schluns, M. Dietel, and I. Petersen. 2001. Overexpression of c-erbB2 protein correlates with disease-stage and chromosomal gain at the c-erbB2 locus in non-small cell lung cancer. Eur. J. Cancer 37:1089-1095.

15.Franklin, W.A., R. Veve, F.R. Hirsch, B.A. Helfrich, and P.A. Bunn, Jr. 2002. Epidermal growth factor receptor family in lung cancer and premalignancy. Semin. Oncol 29:314.

Received 20 November 2002; accepted 26 March 2003.

Address correspondence to Charles $R$. Buck, Oridis Biomed, Elisabethstrasse 84, 8010, A-8010, Graz, Austria. e-mail: buck @oridis-biomed.com 